\title{
A Need for Bandwidth Limitations in Electron Microscopes
}

\author{
Edgar Voelk1 ${ }^{1}$, Peter Tiemeijer ${ }^{2}$ \\ ${ }^{1}$ FEI Company, Hillsboro, Oregon, USA. \\ ${ }^{2}$ FEI Company, Eindhoven, Netherlands.
}

In light-optical microscopes, numerical apertures (apertures in the back focal plane of the objective lens) are an integral part. Often, these apertures can be adjusted continuously, e.g., in single lens reflex (SLR) cameras. In electron microscopy however, and despite much larger magnification ranges, there is only a limited selection of fixed apertures. This is due a) to the pm wave length of the electrons resulting in very small scattering angles from objects with lattice constants in of about 100-400pm and b) to manufacturing limitations and contamination issues as apertures with decreasing hole sizes tend to contaminate easily. While many transmission electron microscopes have a selection of only 4-6 apertures, they offer tremendous magnification ranges. Yet, for most magnifications, suitable numerical apertures are not available. This actually will either become or already is a problem.

To point out the problem, let's start at the highest magnification on, e.g., a spherical aberration-corrected Transmission Electron Microscope (TEM). Assume further the image is acquired (continuously or live) with a charge coupled device (CCD) camera; its live Fourier Transform (FT) is displayed next to the live image. The sample is crystalline and reasonably oriented and the diffraction spots are well visible in the live FFT. By decreasing the magnification of the microscope, a larger area appears in the live image; while in the live Fourier transform the diffraction spots move away from the centre of the image towards the edge of the FT. As they approach that edge with decreasing magnification, they generally seem to disappear. However, if the CCD camera is used with its highest binning factor, let's say 8, a different story evolves. Assume that at highest mag all diffraction spots are well visible in the live FT. As the magnification decreases, the spots walk away from the centre, but as the spots approach the edge of the FT (so-called Nyquist limit), they do not disappear; instead with decreasing magnification, they disappear from one side of the FT and enter the FT from the opposite side. As the magnification decreases further, these spots then actually move towards the centre of the FT. Such spots cause severe artefacts, e.g., physically impossible crystallographic structures.

Inserting a numerical aperture is the obvious choice to re-establish an artefact free image. However, the use of such an aperture only postpones the inevitable artefacts that instantly reappear by further lowering the magnification beyond to where no more smaller apertures are available. This issue holds for any object as any object in an electron microscope can be described via a Fourier series.

Currently, this problem can often be ignored for two reasons: the modulation transfer function (MTF) of the CCD camera and noise in conventional images: close to the Nyquist limit the MTF of a CCD camera is very low $(<<10 \%)$ and together with a limited signal/noise ratio $(\mathrm{S} / \mathrm{N}), \mathrm{CCD}$ cameras (and film for that matter) act like a numerical aperture that automatically adapts to magnification changes.

Recent improvements of data acquisition systems (both data averaging and new detectors that count single electrons), improve the S/N ratio and the MTF. Under such circumstances, the very convenient numerical aperture feature of current CCD cameras will be lost and other means of suppressing information beyond the Nyquist limit need be established. Possible methods might include limiting the 
bandwidth of the electron wave exiting the sample by either moving the sample itself during data acquisition (e.g., via Piezo elements) or by moving the electron beam (after the sample for TEM or before the sample for STEM). Both cases could be achieved by use of either existing or additional deflectors depending on the microscope. Moving the detector during acquisition is another method.

At present, the aforementioned issue already is a significant problem in the area of electron holography, specifically for medium and low magnifications. As described in [1,2], a clear bandwidth limitation is required to separate the so-called sideband from the autocorrelation. Since at medium/low magnifications no standard diffraction aperture is small enough (these apertures are in the wrong plane for low-magnification holography anyway), the reliability of medium/low magnification holography data becomes questionable. An example of such a problematic situation is shown in Figure 1. On the left side, an electron hologram with a field of view of 10um is shown and on its right hand side the corresponding FT is displayed. In the FT the overlap of the autocorrelation and both sidebands is rather obvious. A bandwidth limiting aperture would be required to alleviate the issue, but is not an option.

It is hoped that in the future the possibility of bandwidth limiters becomes a reality - not only for the purpose of electron holography but also to correctly deal with the vastly improved capabilities (both in software and hardware) for future generations of detectors.

\section{References:}

[1] E.N. Leith and J. Upatnieks, JOSA, Vol. 52, Number 10, (1962), p. 1123-1128.

[2] "Introduction to Electron Holography", E. Voelkl, L.F. Allard, D.J. Joy, Kluwer Academic / Plenum Publishers 1999.

[3] D Tang and S Lazar are thanked for their support.
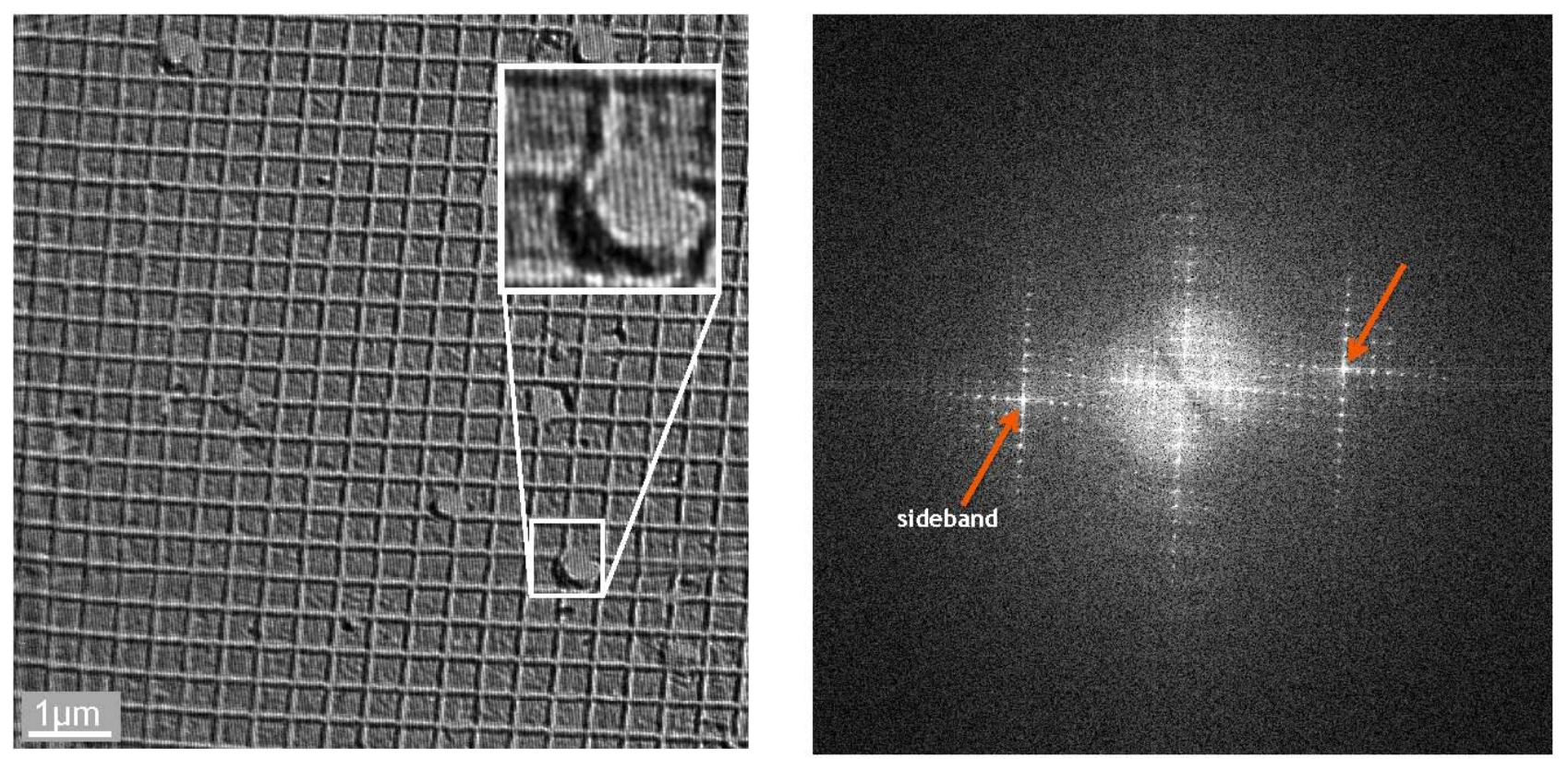

Figure 1. Left: Hologram at low magnification with a field of view of $9 \mathrm{x} 9 \mu \mathrm{m}$. Right: The Fourier transform indicates a significant overlap between the sideband and autocorrelation (center area). No bandwidth limitation (numerical aperture) is available at this point thus violating requirements of [1]. Images were taken on a Titan operated at $300 \mathrm{kV}$ in Lorentz mode (optimized for a large field-of-view). 